\title{
New strategy for monitoring targeted therapy: molecular imaging
}

This article was published in the following Dove Press journal:

International Journal of Nanomedicine

30 September 2013

Number of times this article has been viewed

\section{Fei-Fei Teng \\ Xue Meng \\ Xin-Dong Sun \\ Jin-Ming Yu}

Department of Radiation Oncology,

Shandong Cancer Hospital and

Institute, Shandong University, Jinan,

People's Republic of China
Correspondence: Jin-Ming Yu

Department of Radiation Oncology,

Shandong Cancer Hospital and Institute,

Shandong University, Jinan,

People's Republic of China

Tel +86 531 87984729

Fax +86 53। 87984079

Email jn7984729@public.jn.sd.cn
Abstract: Targeted therapy is becoming an increasingly important component in the treatment of cancer. How to accurately monitor targeted therapy has been crucial in clinical practice. The traditional approach to monitor treatment through imaging has relied on assessing the change of tumor size by refined World Health Organization criteria, or more recently, by the Response Evaluation Criteria in Solid Tumors. However, these criteria, which are based on the change of tumor size, show some limitations for evaluating targeted therapy. Currently, genetic alterations are identified with prognostic as well as predictive potential concerning the use of molecularly targeted drugs. Conversely, considering the limitations of invasiveness and the issue of expression heterogeneity, molecular imaging is better able to assay in vivo biologic processes noninvasively and quantitatively, and has been a particularly attractive tool for monitoring treatment in clinical cancer practice. This review focuses on the applications of different kinds of molecular imaging including positron emission tomography-, magnetic resonance imaging-, ultrasonography-, and computed tomography-based imaging strategies on monitoring targeted therapy. In addition, the key challenges of molecular imaging are addressed to successfully translate these promising techniques in the future.

Keywords: molecular imaging, targeted therapy, PET, MRI, US, CT

\section{Introduction}

Cancer is a leading cause of death worldwide, accounting for over 7 million deaths in 2008. ${ }^{1}$ Monitoring early response of tumors to therapy is crucial in clinical practice to reduce adverse effects and to save costs, especially with the growing number of alternative treatment regimens, such as targeted therapy, that are only effective in select subgroups of patients. Targeted therapy is becoming an increasingly important component in the treatment of cancer. Currently, twelve monoclonal antibodies (mAbs) and twelve tyrosine kinase inhibitors (TKIs) have been approved by the Food and Drug Administration for the treatment of cancer. ${ }^{2}$ The traditional approach to monitor treatment through imaging has relied on assessing the change of tumor size using refined World Health Organization criteria and more recently the Response Evaluation Criteria in Solid Tumors (RECIST). ${ }^{3}$ However, these criteria, which are based on the change of tumor size, show some limitations for monitoring targeted therapy.

Targeted therapy is designed to interfere with specific aberrant biological pathways involved in oncogenesis and angiogenesis, which is in contrast to the generalized cytotoxic effects of standard chemotherapy. The effects of the new targeted therapy, such as angiogenesis inhibitors and antivascular therapies, are more complex. Necrosis and cavitation without a change in size are frequently observed over a short period of 
time. With these newer treatments, lack of progression may be associated with a good improvement in outcome, even in the absence of major shrinkage of tumors as evidenced by partial response or complete response. ${ }^{4,5}$ First, the stable disease (SD) in RECIST was defined as the sum of onedimensional measurements of the greatest diameter of the tumor, which has no change or has a reduction less than $30 \%$ (SD-/0) or has an increase of less than $20 \%$ (SD+). ${ }^{6}$ It was reported that patients with TKI therapies in the SD-/0 group had significantly better survival than those in the SD+ group, but the overall survival (OS) of the SD+ group was similar to that of progressive disease (PD) group. These findings indicated that the SD group patients could be divided into different subgroups based on the change of tumor size and on a more effective surrogate endpoint, with a relatively short time interval; this is necessary to monitor the efficacy of the treatment course. In addition PD, defined as the sum of the one-dimensional measurements of the greatest diameter of the tumor, has an increase of at least 30\% in RECIST; the PD always means the timing to change therapeutic regimens. However, for the patients using targeted therapies, particularly the therapies with inhibitors of promoter, with asymptomatic PD, continued use of targeted therapies is recommended and complete termination of treatment in these patients often causes severe "disease flare." All of these factors suggest that targeted therapy is effective even for patients with PD, and thus the applicability of the RECIST criteria is doubted in evaluating targeted therapy. Therefore, the effect of targeted therapy is often underestimated by RECIST criteria based on tumor size.

To solve these problems, some new methods may be able to identify critical molecular tumor targets involved in proliferation, differentiation, cell death and apoptosis, angiogenesis, immune recognition, invasion, and metastasis. Genetic alterations are identified with prognostic and predictive potential concerning the use of molecularly-targeted drugs. In addition, considering the invasive limitations and the issue of expression heterogeneity, molecular imaging is a more attractive and better qualified new method because it enables noninvasive whole body quantitative imaging of targeted drugs at superior spatial and temporal resolution and sensitivity. ${ }^{7-9}$ Molecular imaging is based on the concept that diagnostic tracers will become concentrated in specific areas because of their interaction with molecular species that are distinctly present in a diseased state. Current molecular imaging includes positron emission tomography (PET), magnetic resonance imaging (MRI), ultrasonography (US), and computed tomography (CT). In this review, we focus on the different kinds of molecular imaging used in monitoring targeted therapy and address the key challenges involved in successfully translating promising molecular imaging in the future.

\section{Pathology}

Currently, only in genotype-selected patients who are identified by pathology, could targeted therapies be used as first-line treatments. Not all patients respond to targeted therapies, but the patients who harbor activating mutations in the target genes are clinically distinct entities who present with a much better prognosis. Several targeted therapies have been approved by National Comprehensive Cancer Network. For example, patients with epidermal growth factor receptor (EGFR) mutations, most frequently exon 19 deletions and exon 21 point mutation L858R may respond to EGFR-TKI, like gefitinib or erlotinib. Conversely, T790M or mesenchymal-epithelial transition factor (MET) amplification may cause acquired resistance. In addition, crizotinib, originally in development as a Met inhibitor, is also a potent anaplastic lymphoma kinase gene inhibitor, which results in a rapid and dramatic response for non-small- cell lung cancer (NSCLC) patients based on anaplastic lymphoma kinase gene rearrangements. ${ }^{10}$ As a marker of aggressive disease, human EGFR (HER) 2 overexpression is an independent predictor of breast cancer-related survival. ${ }^{11}$ For patients with HER2-positive breast cancer, trastuzumab is recommended for first-line treatment. ${ }^{12}$

Molecular profiling of tumors is mainly done on biopsied samples. Although the present review focuses more on the biomarker utility of these genes and less on the technicalities of their measurement, we must emphasize that the acquisition of adequate biopsy material remains problematic. Immunohistochemistry of the gene product proteins is the predominant method used to detect gene expressions, although fluorescent in-situ hybridization, enzyme-linked immunosorbent assay, and ribonucleic acid-binding assays are also used increasingly. ${ }^{2}$ The predictive power of pathology may be limited due to the following aspects: (1) biopsies are invasive and not frequently repeated, and target expressions can vary during therapy due to clonal selection; and (2) the predictive power may be limited due to intratumoral expression heterogeneity and the discordance of molecular target expressions between primary tumors and matched metastases. ${ }^{13,14}$ Because of the limitations of tissue specimens, elevated plasma levels or serum cell free deoxyribonucleic acid (DNA) levels in lung cancer patients have been previously reported as a new testing method. ${ }^{15}$ However, serum 
cell free DNA-associated mechanisms have not yet been clarified, and the mutation status in the plasma or serum is not always consistent with that in tissues. Recently, Zhao et $\mathrm{al}^{16}$ demonstrated that plasma DNA may be a viable alternative to tumor samples for the detection of EGFR mutations, which requires further evaluation.

However, molecular imaging as a new paradigm is noninvasive and allows serial investigations, which are essential for monitoring the therapeutic efficacy of drugs during the whole treatment course. In addition, molecular imaging (as a well reproducible, quantitative method) can be safely performed for any lesion and be repeated multiple times; it permits the evaluation of an entire tumor, provides information related to regional heterogeneity in a given tumor, and thus reduces the sampling variability due to intratumor heterogeneities. ${ }^{17}$

\section{Positron emission tomography (PET)}

PET relies on the administration of exogenous probe molecules labeled with radioisotopes (tracers) that emit positrons over time, which can detect therapy-induced molecular and cellular changes that occur earlier than anatomical changes, such as decreases in tumor volume. PET can be done repeatedly to monitor therapeutic efficacy in the same patient during therapy. In addition, most modern PET scanners are equipped with $\mathrm{CT}$ scanners that give anatomical landmarks for in vivo biochemistry. Because of these advantages, PET represents a promising avenue for monitoring disease progression and response to therapy when compared with conventional imaging modalities employed by RECIST. As a common approach, PET molecular imaging can be classified as a surrogate and direct molecular imaging, which is based on the different radiolabeled probes used and on different endogenous molecular processes. ${ }^{18}$

\section{Surrogate molecular imaging}

Surrogate molecular imaging is generally related to the imaging of specific molecules and cellular processes, such as glycose metabolism and cell proliferation. Tumor response to therapy is an extremely complicated process, even though therapy might aim at a particular target or specific pathway. The glucose analog 2'-deoxy-2'-(18F)fluorodeoxyglucose (FDG) is a surrogate marker for glucose metabolism and is the most commonly used radiopharmaceutical for PET. Unlike glucose-6-phosphate, ${ }^{18}$ F-FDG-6-phosphate is not a substrate of glucose-6-phosphate isomerase and does not undergo further metabolism in the glucose pathway. ${ }^{18} \mathrm{~F}-\mathrm{FDG}$ is therefore trapped within cells. ${ }^{19}$ In addition, $3^{\prime}$-deoxy-39-
(18F)fluorothymidine (FLT) is a pyrimidine analog that, after uptake into the cell, is phosphorylated by thymidine kinase 1 into ${ }^{18} \mathrm{~F}$-FLT monophosphate, causing intracellular sequestration of radioactivity. Thymidine kinase 1 is a principal enzyme in the salvage pathway of DNA synthesis. ${ }^{18} \mathrm{~F}$-FLT uptake, therefore, reflects cellular proliferation. Currently, ${ }^{18} \mathrm{~F}$-FDG PET and ${ }^{18} \mathrm{~F}$-FLT PET are more clinically advanced than several other molecular imaging approaches for treatment monitoring. ${ }^{18} \mathrm{~F}$-FDG and ${ }^{18} \mathrm{~F}$-FLT accumulation during the treatment of many cancers significantly correlates with prolonged survival, thereby fulfilling requirements for predictive biomarkers. In recent years, a variety of literature has reported that ${ }^{18} \mathrm{~F}$-FDG PET and ${ }^{18} \mathrm{~F}$-FLT PET could predict the benefits of TKIs in NSCLC patients (Table 1). In a Phase II trial, Zander et $\mathrm{al}^{20}$ demonstrated that an early (1 week after treatment) metabolic ${ }^{18} \mathrm{~F}-\mathrm{FDG}$ response had significantly longer progression free survival (PFS) and OS in advanced NSCLC patients administered with erlotinib. Early ${ }^{18}$ F-FLT response predicted significantly longer PFS rather than OS. A small proportion of patients without the EGFR mutation benefitted from erlotinib treatment, which can be identified by early ${ }^{18} \mathrm{~F}$-FDG PET. This benefit may result from insufficient efficacy of EGFR mutation testing and/or of antiwild-type EGFR effects of erlotinib. ${ }^{20}$ Kahraman et $\mathrm{al}^{21}$ showed results that metabolically active volume measurement in early ${ }^{18} \mathrm{~F}$-FLT PET and late ${ }^{18} \mathrm{~F}$-FDG PET may have an additional predictive value in monitoring response in patients with advanced NSCLC treated with erlotinib. Therefore, a proportion of patients who benefit from erlotinib treatment without having any genetic mutations detected might be identified by early ${ }^{18} \mathrm{~F}$-FDG PET. ${ }^{20}$

${ }^{18}$ F-FLT PET has been more promising in measuring response to targeted therapy under some select conditions, such as in patients with a higher probability of mutations. The role of ${ }^{18}$ F-FLT PET might be gaining importance in those patients who are Asian, nonsmoking, and have adenocarcinoma histology, as well as those who are more likely to carry mutations. ${ }^{22}$ Furthermore, ${ }^{18} \mathrm{~F}$-FDG PET relies on metabolic differences in glucose use, but it should be noted that not all cancers undergo energy metabolism through glucose metabolism. Besides, glucose accumulates both in tumor and in inflammatory cells, while the latter are often induced by irradiation. It was reported that ${ }^{18} \mathrm{~F}$-FLT PET may discriminate tumor from esophagitis more effectively than ${ }^{18} \mathrm{~F}-\mathrm{FDG}$ PET based on pathology evaluation. ${ }^{23}$ At the same time, different quantitative parameters for ${ }^{18} \mathrm{~F}$-FDG PET and ${ }^{18} \mathrm{~F}$-FLT PET have been used to evaluate clinical benefit of TKIs. Kahraman et $\mathrm{al}^{21}$ described a metabolic response on the reduction of 
Table I Recent literature about the use of ${ }^{18} \mathrm{~F}$-FDG PET and ${ }^{18} \mathrm{~F}$-FLT PET on predicting the benefits of TKIs in patients with lung cancer

\begin{tabular}{|c|c|c|c|c|c|}
\hline Authors & Drug & $\begin{array}{l}\text { Number } \\
\text { of patients }\end{array}$ & Probe & Parameters & Results \\
\hline Zander et $\mathrm{al}^{20}$ & Erlotinib & 34 & FDG, FLT & $\begin{array}{l}\text { Changes in FDG and FLT uptake } \\
\text { after I week and } 6 \text { weeks of erlotinib } \\
\text { treatment }\end{array}$ & $\begin{array}{l}\text { Early FDG/FLT response was correlated } \\
\text { with PFS; early FDG response was } \\
\text { correlated with OS }\end{array}$ \\
\hline Mileshkin et a ${ }^{35}$ & Erlotinib & 51 & FDG, FLT & $\begin{array}{l}\text { Changes in FDG and FLT uptake after } \\
\text { day } 14 \text { and day } 56 \text { of erlotinib treatment }\end{array}$ & $\begin{array}{l}\text { Early FDG/FLT response was correlated } \\
\text { with PFS; early FDG was correlated with OS }\end{array}$ \\
\hline Takahashi et al ${ }^{36}$ & Gefitinib & 20 & FDG & $\begin{array}{l}\text { Changes in FDG uptake after } \\
\text { two days of gefitinib treatment }\end{array}$ & $\begin{array}{l}\text { FDG response was not statistically } \\
\text { associated with PFS }\end{array}$ \\
\hline Bengtsson et $\mathrm{a}^{37}$ & Erlotinib & 125 & FDG & $\begin{array}{l}\text { Changes in FDG uptake after } \\
2 \text { weeks of erlotinib treatment }\end{array}$ & $\begin{array}{l}\text { Reduction of maximum standardized } \\
\text { uptake value by at least } 35 \% \text { was } \\
\text { predictive of survival }\end{array}$ \\
\hline Scheffler et $\mathrm{al}^{24}$ & Erlotinib & 40 & FDG, FLT & $\begin{array}{l}\text { FLT and FDG SUVmax before } \\
\text { erlotinib treatment (baseline) }\end{array}$ & $\begin{array}{l}\text { FDG SUVmax }<6.6 \text { and FLT SUVmax }<3.0 \\
\text { had a significantly better overall survival }\end{array}$ \\
\hline Kahraman et $\mathrm{al}^{26}$ & Erlotinib & 30 & FDG, FLT & Percentage changes of TLG and TLP & $\begin{array}{l}\text { Lower absolute early and late residual } \\
\text { TLG and TLP levels had a significantly } \\
\text { prolonged PFS }\end{array}$ \\
\hline Kobe et $\mathrm{a}^{25}$ & Erlotinib & 30 & FDG, FLT & $\begin{array}{l}\text { I- and 6-week residual FDG and FLT } \\
\text { uptake were measured with different } \\
\text { quantitative standardized uptake values }\end{array}$ & $\begin{array}{l}\text { Nonprogression after } 6 \text { weeks was } \\
\text { associated with a significantly lower early } \\
\text { and late residual FDG uptake }\end{array}$ \\
\hline
\end{tabular}

Abbreviations: FDG, 2'-deoxy-2'-(I8F)fluorodeoxyglucose; PET, positron emission tomography; FLT, 3'-deoxy-39-(I8F)fluorothymidine; TKI, tyrosine kinase inhibitors; PFS, progression-free survival; OS, overall survival; SUVmax, maximum standard uptake value; TLG, total lesion glycolysis in FDG PET; TLP, total lesion proliferation in FLT PET.

${ }^{18} \mathrm{~F}$-FDG and ${ }^{18} \mathrm{~F}$-FLT uptake, regardless of the methods used to calculate the standard uptake value (SUV). Scheffler et $\mathrm{al}^{24}$ reported that the low baseline maximum SUV (SUVmax) of ${ }^{18} \mathrm{~F}$-FDG and ${ }^{18} \mathrm{~F}$-FLT significantly correlated with a better OS. Besides, residual SUVmax and functional tumor volume parameters including total lesion glycolysis in ${ }^{18} \mathrm{~F}$-FDG PET and total lesion proliferation in ${ }^{18} \mathrm{~F}$-FLT PET have also been used to describe response to treatment. ${ }^{25,26}$ Currently, according to the Positron Emission Tomography Response Criteria in Solid Tumors (PERCIST) guidelines, reduction in ${ }^{18} \mathrm{~F}$-FDG uptake of $30 \%$ was defined as a metabolic response, and an increase of $30 \%$ was defined as progression. ${ }^{27}$ Those criteria for $18 \mathrm{~F}-\mathrm{FDG}$ are widely recognized. However, there are still no acknowledged criteria for ${ }^{18} \mathrm{~F}$-FLT or other probes.

Furthermore, recent preclinical studies reported that ${ }^{18} \mathrm{~F}$-FLT PET imaging and ${ }^{18} \mathrm{~F}$-FDG PET imaging are useful tools for early response monitoring of a novel c-Met inhibitor, BAY 853474, in a gastric cancer xenograft model. ${ }^{28}$ Kasper et $\mathrm{al}^{29}$ demonstrated that early SUV changes on ${ }^{18} \mathrm{~F}$-FDG PET may help to discriminate responders from nonresponders and, thus, to decide whether imatinib therapy should be continued. Imatinib, as a $B C R-A B L$ (a gene sequence found in an abnormal chromosome 22 of some people with certain forms of leukemia) and $c$-KIT inhibitor, directly affects the expressions of glucose transporters that can be measured by ${ }^{18}$ F-FDG PET. ${ }^{29}$ For the HER1/HER2 inhibitor PKI-16620 and the Met inhibitor PD032590121, ${ }^{18}$ F-FLT might be a more sensitive pharmacodynamic biomarker than ${ }^{18} \mathrm{~F}-\mathrm{FDG}$, due to the fact that they indicate changes in imaging proliferation with radiolabeled thymidine analogs. ${ }^{30,31}$ More research is warranted to translate ${ }^{18} \mathrm{~F}$-FDG PET or ${ }^{18} \mathrm{~F}$-FLT PET in clinical settings for the routine monitoring of targeted therapy.

In addition to energy metabolism and proliferation, tumor hypoxia has been also reported in association with an aggressive tumor phenotype, poor response to radiotherapy and chemotherapy, increased risk of invasion and metastasis, and worse prognosis. Over the last decade, hypoxia imaging has become applicable by using radiolabeled hypoxia agents together with noninvasive imaging techniques such as PET or single photon emission computed tomography. Nitroimidazole is thought to be a bioreducible group, and is thus a marker of hypoxic tissue. Under hypoxic conditions, the nitro group of nitroimidazole is further reduced under enzymatic catalysis of nitroreductase, followed by decomposition, to form highly reactive intermediates such as free radicals, which can bind to cellular macromolecules and be trapped in the hypoxic cell irreversibly. Several nitroimidazole compounds with different properties and labeled with different PET radionuclides have been described, ${ }^{32}$ such as (18F)fluoromisonidazole ( ${ }^{18} \mathrm{~F}$-FMISO), (18F)fluoroazomycin-arabinofuranoside, (18F) fluoroetanidazole, [18F]fluoroerythronitroimidazole, ${ }^{18} \mathrm{~F}-2-(2-$ nitro-(1)H-imidazol-1-yl)-N-(2,2,3,3,3-pentafluoropropyl)acetamide, and (124I)iodoazomycinarabinoside. 
Currently, although much of the field is still at the preclinical stage, many clinical studies have been performed for PET imaging of hypoxia. Among them, ${ }^{18} \mathrm{~F}$-FMISO is the most extensively studied PET radiotracer of hypoxia. Because hypoxia imparts resistance to treatment, ${ }^{18} \mathrm{~F}-\mathrm{FMISO}$ PET has been used in the treatment of head and neck cancer, and shows potential for guiding radiation therapy to overcome hypoxia-induced resistance. ${ }^{33}$ Much of the recent efforts in the area focus on the bioreducible organic compounds as hypoxia imaging agents. There has been a growing interest in hypoxic selectivity based on ligand receptor interaction, and dual- or multi-modality molecular imaging has also attracted increasing attention. ${ }^{34}$ However, applications for imaging hypoxia on targeted therapy are still in very early stages, which may be promising in monitoring the efficacy of antiangiogenesis-targeted therapies.

\section{Direct molecular imaging}

For direct molecular imaging, probes are needed to direct specific molecular targets like transporters or enzymes. Transporters or enzymes measured by direct molecular imaging should be earlier and more sensitive pharmacodynamic biomarkers used to reflect therapeutic efficacy than either glycolysis or DNA synthesis, which are measured by surrogate imaging. In recent years, many preclinical and clinical studies suggest that direct molecular imaging provides useful methods for monitoring targeted therapy. The basic principles of molecular imaging are specificity and susceptibility, which mean obtaining significantly high signal intensity by the use of minimal amounts of molecular probe. The ideal probe would have the following characteristics: $:^{38}$ (1) the probe should not cause an immune response; (2) the probe should be stable in vivo and not be metabolized before reaching its target; (3) after the completion of its process, the probe should rapidly clear from the circulation and not interfere with the detection of a specific signal; (4) the probe or its metabolites should not be cytotoxic; (5) the size of the probe should be small enough to go through natural biological barriers; and (6) the image signal intensity should be directly proportional with the amount of probe. The direct molecular imaging probes in common use can be assessed by different targeted ligands, mAbs or their fragments, natural peptide ligands or their analogs, TKIs or their analogs, and high-affinity peptides.

\section{Monoclonal antibody}

To enable visualization of a targeted $\mathrm{mAb}$ with a PET camera, the drug should always be labeled with an inert positron emitter. The physical half-lives of the positron emitters should be compatible with the residence time of the targeted drug in the body, which is typically for several days for slow kinetic intact mAbs, and a couple of hours for the fast kinetic small molecules like TKIs. ${ }^{39}$ Very recently, universal procedures were introduced for radiolabeling of intact $\mathrm{mAbs}$ with the long-lived positron emitters, such as iodine- $124\left(\mathrm{t}^{1} / 2=100.3\right.$ hours $),{ }^{40}$ and zirconium- $89\left({ }^{89} \mathrm{Zr}\right.$; $\left.\mathrm{t}^{1} / 2=78.4 \mathrm{~h}\right){ }^{41}$ The first clinical results have indicated that ${ }^{89} \mathrm{Zr}$ can be successfully used to detect HER2-positive breast cancer metastases..$^{42}$ Recently, van der Bilt et al ${ }^{43}$ confirmed that ${ }^{89} \mathrm{Zr}$ PET could be used to monitor tumor vascular endothelial growth factor receptor (VEGFR) A levels as an early biomarker of the antiangiogenic effect of mammalian target of rapamycin inhibitor therapy in an ovarian cancer xenograft model. However, the imaging potential of radioimmuno targeting has been limited by the slow clearance of full-length antibodies from blood, as well as by their poor extravasation and diffusion into the extracellular space. ${ }^{2}$ Furthermore, because of the tumor's enhanced permeability and retention effect, $\mathrm{mAbs}$ accumulate in tumor, which leads to lower specificity.

\section{Monoclonal antibody fragments and pretargeting immunoimaging}

The large size of intact antibodies $(\sim 150 \mathrm{kDa})$ results in slow clearance, high-background signal, and nonspecific accumulation. Fragments and constructs with sizes below the renal filtration threshold, however, clear rapidly, reducing not only background signal but also tumor uptake and accumulation. Technologies based on enzymatic digestion and genetic modifications of antibodies have been used to generate antibody derivatives with improved pharmacokinetic properties. It was reported that in HER2 tumor xenografts, ${ }^{68}$ Ga-DOTA-F(ab')2-trastuzumab PET imaging identifies HER2 downregulation associated with Hsp90 inhibition prior to changes in glycolysis seen using ${ }^{18} \mathrm{~F}$-FDG PET. ${ }^{44}$ Much of the information has been obtained from preclinical studies over the past few years. Clinical studies have been done in limited numbers of patients. Six antibody fragments have been Food and Drug Administration approved, and three of them are used in single photon emission computed tomography. While labeled antibody fragments have higher relative tumor-to-normal-tissue ratios than whole antibodies, the total tumor uptake is low and most of the administered label is excreted. A new pretargeting immunoimaging method has been developed to separate target accumulation and probe attachment, and could enhance tumor-to-background ratios, maximize uptake, and minimize radiation exposure. This is 
achieved with antibodies that bind to both a specific antigen and a secondary molecule with an imaging group, either through another binding region or an attachment. ${ }^{45}$ Such multistep approaches reduce signal background related to slow clearance of antibodies by separating the slow distribution of the antibody from the fast decay of radionuclides. ${ }^{2}$

\section{Natural peptide ligands or their analogs}

Unlike mAbs or their fragments, natural peptide ligands show rapid systemic clearances and higher tumor-to-organ ratios by the smaller molecules. In 1996, Cuartero-Plaza et $\mathrm{al}^{46}$ imaged EGFR in squamous lung carcinoma with radiolabeled (131I) epidermal growth factor, which had shown that radiolabeled natural ligands can be used for receptor imaging in vivo. However, the imaging potential was limited by the high liver uptakes ratios; at the same time, adverse physiological reactions restrict the administration of larger amounts of the ligands. Shortlived positron emitters could be used in radiolabeling the ligand because of the rapid systemic clearances, such as Ga ( $\mathrm{t}^{1} / 2=68$ minutes). Recently, a clinical study reported the use of ${ }^{61} \mathrm{Cu}$, as the radiolabel is desirable for small proteins such as K-3-VEGF(121), which has a much higher beta $(+)$ branching ratio than the commonly used ${ }^{64} \mathrm{Cu}(62 \%$ versus $17 \%$ ), thereby offering stronger signal intensity and lower tracer dose for PET imaging. ${ }^{47}$ However, to prevent natural ligands from undergoing rapid internalization and degradation after binding, new antagonistic analogs must be developed. Labeling with radiometals instead of radiohalogens is recommended. ${ }^{2}$

\section{Tyrosine kinase inhibitors (TKIs) or their analogs}

In contrast to the radiolabeling of mAbs, radiolabeling of small molecules $(<1 \mathrm{kDa})$ like TKIs is much more challenging in the following aspects. First, the intracellular kinases are the primary targets of TKIs. It is better to use labeling strategies that end up with exactly the original TKI chemical structure in which a cold carbon or fluorine atom has been substituted by ${ }^{11} \mathrm{C}$ or ${ }^{18} \mathrm{~F}$, respectively, instead of the addition of an inert radioactive element, to make sure that the radiolabeled TKI is small enough to go through the cell membrane. Second, TKIs are different from mAbs, which are normally metabolically stable in the blood circulation, and where radioactive metabolites might be formed. To conquer this problem, it might be interesting to label a TKI at different positions if possible, and to select the most suitable candidate tracer. ${ }^{39}$ Third, TKIs must be lipophilic enough to diffuse through cellular membranes to reach the binding sites, but a higher lipophilicity increases the probability of hepatobiliary excretion, leading to high radioactivity concentrations in the abdomen. ${ }^{48}$ In a study of Memon et al, ${ }^{49}$ responders and nonresponders to erlotinib show different uptakes of ${ }^{11} \mathrm{C}$-erlotinib PET, even though there were only 13 patients. ${ }^{11} \mathrm{C}$-labeled $4-\mathrm{N}$-(3-bromoanilino)-6,7dimethoxyquinazoline, a positron-emitting analog of the EGFR-TKI PD153035, was developed as a noninvasive imaging biomarker for tumor EGFR status using PET. ${ }^{50} \mathrm{It}$ is vital that novel TKI-PET tracers are designed with care, not only retaining the native chemical structure, but also successfully passing thorough metabolite analyses and biodistribution studies in relevant animal models before performing clinical studies. ${ }^{51}$

\section{High-affinity peptide and affibody}

Scaffold high-affinity proteins, such as affibody molecules, are a new and promising class of probes used for in vivo imaging. The use of small radiopeptides, based on natural ligands or their analogs, provides rapid targeting kinetics with better imaging sensitivity and specificity than intact antibodies. However, their rather short biological half-lives and the risk of agonist effects significantly limit their applicability. Several classes of scaffold protein-based radiolabeled probes have a high potential for in vivo molecular imaging, including affibody molecules, cystine knot peptides, and nanobodies. ${ }^{52}$ Several selected therapeutic targets have been developed that affibody molecules exhibit high-affinity binding to, such as HER2, EGFR, insulin-like growth factor 1 , and platelet-derived growth factor receptor B. ${ }^{53-55}$ AntiEGFR affibody-based molecular probes, ${ }^{64} \mathrm{Cu}$-DOTA-Cys$Z_{\mathrm{EGFR}}: 1907$ and Cy5.5-Cys- $Z_{\mathrm{EGFR}}: 1907$, were successfully prepared and found to show rapid tumor targeting ability and good tumor imaging contrast even at 1 hour after injection in the EGFR-expressing A431 tumor xenograft model. ${ }^{56,57}$ In addition, radiolabeled affibody molecules have been used to monitor the degradation of HER2 in response to inhibition of HSP90, ${ }^{58,59}$ showing that they might be used not only for patient stratification, but also for therapy monitoring. In a new animal study, the results demonstrated that small differences in the composition of a binding site can influence the biodistribution of HER2-binding affibody molecules, including uptake in normal tissues. Recently, an increasingly appreciated strategy by using bispecific constructs has been shown to increase the efficacy and selectivity in imaging and therapy. Additional future studies should be conducted in vivo to further investigate the therapeutic effects of the bispecific constructs. 
PET shows more potential in imaging the functional status of a tumor, which is important for targeted therapy in limited tumor size changes based on RECIST. Besides PET, MRI, US, and CT are most frequently used in the clinic for molecular imaging.

\section{Magnetic resonance imaging (MRI) and magnetic resonance spectroscopy (MRS)}

MRI is widely used clinically to assess tumor growth and for response evaluation, which generally measures the magnetic relaxation properties of protons. MRI has no limitation for tissue penetration, does not use ionizing radiation, and offers higher resolution and soft tissue contrast. These advantages make MRI highly desirable for molecular imaging.

By far, the most widely used MRI contrast agents are those based on the paramagnetic gadoliniumion $(\mathrm{Gd})$, which are often chelated to low molecular weight ligands, such as diethylene triamine pentacetate acid and 1,4,7,10tetraazacyclododecane-1,4,7,10-tetraacetic acid, which are used to reduce toxicity ${ }^{60}$ and are loaded onto nanocarriers to enhance detection sensitivity. MRI has been used for molecular imaging of tumor angiogenesis by targeting integrin expression. The first MRI approach for imaging integrin $\alpha v \beta 3$ expression on tumor angiogenesis was demonstrated by Sipkins et al. ${ }^{61}$ As well, Boles et a ${ }^{62}$ used integrin $\alpha v \beta 3$-targeted gadolinium $\left(\mathrm{Gd}^{3+}\right)$ chelate-containing perfluorocarbon nanoparticle to image integrin $\alpha v \beta 3$ expression in a murine melanoma tumor model at a clinical field strength (3 Tesla).

The nano constructs, $\mathrm{Gd}^{3+}$-containing micelles, liposomes, or high-density lipoprotein-like nanoparticles ${ }^{63}$ can enable detection in the picomolar range (particle concentration), and can therefore enable visualization with MRI of sparse binding sites while improving magnetic resonance sensitivity for tumor angiogenesis imaging. Mulder et al, ${ }^{64}$ using integrin $\alpha v \beta 3$-targeted bimodal liposomes in a tumor mouse model with MRI showed a very good correlation with microvessel density, and demonstrated that molecular MRI can be used to noninvasively measure the efficacy of angiogenesis inhibitors during the course of therapy. MRI approaches using magnetic nanoparticles have recently been validated for the examination of tumor vascularity in vivo. Guimaraes et a ${ }^{65}$ demonstrated noninvasive, in vivo antiangiogenic monitoring using MRI of mammalian target of rapamycin inhibition with ultrasmall super paramagnetic iron oxide nanoparticles. Another promising approach based on magnetic nanoparticles is to use nanoparticles targeted against markers overexpressed on tumor cells. Abdolahi et al ${ }^{66}$ used superparamagnetic iron oxide nanoparticle attached to an antibody (J591) that binds to the extracellular domain of the prostate-specific membrane antigen (PSMA), to specifically enhance the contrast of PSMA-expressing prostate cancer cells. MRI and cell uptake experiments demonstrated the high potential of the synthesized nanoprobe as a specific MRI contrast agent for the detection of PSMA-expressing prostate cancer cells.

An attractive emerging method is the expression of tailored artificial peptides, which makes use of the chemical shift sensitivity of chemical exchange saturation transfer (CEST)-MRI. ${ }^{67}$ CEST-MRI has been used to monitor enzymatic activity, ${ }^{68}$ as well as to measure the contrast generated by poly-L-lysine alongside T2 contrast generated by superparamagnetic iron oxide particles when mixed together in phantoms ${ }^{69}$ Paramagnetic CEST agents are ideally suited for molecular imaging applications because one can switch the contrast on and off as desired simply by adjusting the pulse sequence parameters. Liposome-based CEST agents have shown great sensitivity and potential for molecular MRI. ${ }^{70}$

While MRI uses the signal from hydrogen protons to form anatomic images, proton MRS uses this information to determine the concentration of brain metabolites such as $\mathrm{N}$-acetyl aspartate (NAA), choline (Cho), creatine (Cr), and lactate in the tissue examined. MRS techniques are based on the principle that it is possible to detect radiofrequency signals generated by magnetic nuclear spins of magnetic resonance-active nuclei such as ${ }^{1} \mathrm{H},{ }^{31} \mathrm{P},{ }^{13} \mathrm{C}$, and ${ }^{19} \mathrm{~F}$ processing in an external magnetic field $\mathrm{B} 0 .{ }^{71}$ It was reported that a decrease of total Cho and phosphatidyl choline (PC) was observed following treatments against molecular targets such as mitogen activated protein kinase ${ }^{72}$ and Bcr-Abl tyrosine kinase. ${ }^{73}$ Thus, the therapeutic responses can be monitored by MRS. However, PC levels were also found to increase following histone deacetylase inhibition. ${ }^{74}$ Therefore, a decrease of total Cho or PC cannot always be associated with response, as this will depend on the target selected. Currently, MRS methods are being explored to detect the uptake and distribution of drugs labeled with ${ }^{19} \mathrm{~F},{ }^{13} \mathrm{C}$, or ${ }^{1} \mathrm{H} .{ }^{75}$ In addition, MRI and MRS provide indirect ways of assessing tumor oxygenation. Blood oxygenation level-dependent MRI contrast is generated by a change in local deoxyhemoglobin concentration $\mathrm{Hb}$. Blood oxygenation level-dependent contrast has been examined as a potential means to indirectly evaluate changes in tumor oxygenation in vivo. ${ }^{76}$

Sensitivity and spectral resolution continue to be limiting factors of MRS techniques. MRS methods may provide an understanding of the effects of down-regulating 
specific targets on downstream changes in physiology and metabolism, which are a rich source to mine for noninvasive biomarkers associated with molecular targets.

\section{Ultrasonography (US)}

Ultrasound imaging is inexpensive, widely available, and completely noninvasive. Contrast-enhanced ultrasound has clinical applications in echocardiography and hepatology for imaging liver cancer. With a limiting size of several micrometers, microbubbles (purely intravascular contrast particles) allowed recirculation in the bloodstream. Targeted imaging with microbubbles is performed by targeting the bubble surface with suitable ligands, which are molecules with a specific affinity for the biomarkers of a disease. Bubbles are injected intravenously, circulate, bind to the target receptors on vascular endothelium, and accumulate at the disease sites. Echo from targeted bubbles allows visualization of the biomarker pattern. ${ }^{77}$

Although much of the field is still at the preclinical stage, clinically translatable microbubbles have been devised for tumor vasculature targeting, and a successfully completed small-scale exploratory clinical trial has been conducted. It has been shown that microbubbles carrying cyclic arginine-glycine aspartic acid peptide ${ }^{78}$ and knottin peptides ${ }^{79}$ were feasible for ingtarget integrin $\alpha v \beta 3$ in tumor models. Microbubble targeting of the angiogenic marker VEGFR-2 has been targeted by microbubble with anti-VEGFR-2 antibody ${ }^{80}$ and a single-chain VEGF construct. ${ }^{81}$ A more recent concept is multitargeting, which is the targeting against more than one molecular marker with microbubbles carrying two or three ligands, and which has improved visualization of the tumor vasculature. ${ }^{82}$ Multitargeting may be of importance, particularly in early tumor detection, when different markers are expressed at different time points along the tumor development. Using molecularly targeted microbubbles, Deshpande et al ${ }^{83}$ evaluated the level of expression of three angiogenic markers - integrin $\alpha v \beta 3$, endoglin, and VEGFR-2 - on tumor vascular endothelial cells in vivo during tumor growth. They concluded that targeted contrast-enhanced US imaging allows noninvasive in vivo assessment of the expression levels of integrin $\alpha v \beta 3$, endoglin, and VEGFR-2, which vary during tumor growth in subcutaneous cancer xenografts. In addition, targeting of molecular angiogenic markers shows potential to monitor the efficacy of drug therapy.

However, the dependence on the skill of the operator and limited depth penetration limits the use of ultrasound. Moreover, not all regions of the body are accessible with ultrasound (such as lung, bone, and brain in adults).

\section{Computed tomography (CT)}

The relatively low cost of CT, as well as fast image acquisition and high spatial resolution, also constitute factors that favor its utilization in the clinic. The development of molecular CT imaging agents is still in a very early stage, and only a few agents have shown promise in in vivo models. The polymercoated bismuth sulfate nanoparticle was one of the first CT nanoparticle agents reported by Rabin et al.$^{84}$ In the following year, an iodinated nanoparticle dispersed with surfactant was reported. ${ }^{85}$ Recently, gold nanoparticles have been shown to match or exceed the performance of conventional iodinated contrast agents under conditions relevant for $\mathrm{CT} .{ }^{86}$ Targeted gold nanoparticles for cancer imaging have been described, and their use in in vitro cell samples has been demonstrated. ${ }^{87}$ However, a major obstacle that CT has and will be confronted with is sensitivity. In addition, because of the intrinsic principle of CT measuring X-ray absorption - a method used to increase $\mathrm{CT}$ attenuation of the contrast agent at the target site other than through a sheer increase in concentration - does not seem feasible.

\section{Conclusion}

Conventional structural imaging modalities can offer images with exquisite spatial resolution within seconds or minutes, but they share the limitation of not being able to detect lesions until the structural changes in the tissue are large enough to be detected by these imaging technologies. As a new method, molecular imaging shows more potential than conventional imaging in the following aspects, especially for targeted therapy: (1) functional imaging, like ${ }^{18} \mathrm{~F}-\mathrm{FDG}$ PET and ${ }^{18}$ F-FLT PET, can image the functional status of tumor in glycolysis and DNA synthesis, which may be the main response for cytostatic target therapies, especially in the early treatment stage; (2) imaging the target receptor could reflect the changes of the therapy-acting peptide or kinase directly; and (3) another promising advantage for molecular imaging is the fact that it is a quantitative measurement, which could objectively monitor the therapy response.

In addition, though detecting gene status is currently the standard for patient stratification and for selecting advantage groups for targeted therapy, pathology is not feasible for sequential monitoring therapy because of its invasive limitations, tumor heterogeneity, and variable gene mutation status during therapy. Hence, molecular imaging could be a viable substitute method conquering all these problems.

Molecular imaging has come a long way with PET-based imaging methods in the past decade. MR-, US-, and CT-based molecular imaging techniques have emerged more recently 
and show promise to become viable complements in living animals and humans. The future of molecular imaging is limited in terms of sensitivity, specificity, and clinical translatability. The sensitivity is highly dependent on the particular contrast agent used, and the specificity is dependent on the imaging signal, as a disease process could be improved by combining two or more modalities into a multimodal approach, such as a PET/MRI or MRI/US fusion, where each modality informs about a different characteristic of the pathologic condition. Furthermore, most of the approaches need much more research to translate into the clinical setting and to gain Food and Drug Administration approval.

\section{Acknowledgments}

This work was supported by The National Natural Science Foundation (81201827).

\section{Disclosure}

The authors report no conflicts of interest in this work.

\section{References}

1. Hoogerwerf MA, Ninaber MK, Willems LN, Kaptein AA. "Feelings are facts": illness perceptions in patients with lung cancer. Respir Med. 2012;106(8):1170-1176.

2. Tolmachev V, Stone-Elander S, Orlova A. Radiolabelled receptor-tyrosine-kinase targeting drugs for patient stratification and monitoring of therapy response: prospects and pitfalls. Lancet Oncol. 2010;11(10):992-1000.

3. Eisenhauer EA, Therasse P, Bogaerts J, et al. New response evaluation criteria in solid tumours: revised RECIST guideline (version 1.1). Eur J Cancer. 2009;45(2):228-247.

4. Ratain MJ, Eckhardt SG. Phase II studies of modern drugs directed against new targets: if you are fazed, too, then resist RECIST. J Clin Oncol. 2004;22(22):4442-4445.

5. Rosner GL, Stadler W, Ratain MJ. Randomized discontinuation design: application to cytostatic antineoplastic agents. J Clin Oncol. 2002;20(22):4478-4484.

6. Zhang J, Huang Y, Li X, et al. The impact of tumor size change after target therapy on survival: analysis of patients enrolled onto three clinical trials of advanced NSCLC from one institution. Onco Targets Ther. 2012;5:349-355.

7. van Dongen GA, Visser GW, Lub-de Hooge MN, de Vries EG, Perk LR. Immuno-PET: a navigator in monoclonal antibody development and applications. Oncologist. 2007;12(12):1379-1389.

8. van Dongen GA, Vosjan MJ. Immuno-positron emission tomography: shedding light on clinical antibody therapy. Cancer Biother Radiopharm. 2010;25(4):375-385

9. de Vries EG, Oude Munnink TH, van Vugt MA, Nagengast WB. Toward molecular imaging-driven drug development in oncology. Cancer Discov. 2011;1(1):25-28.

10. Ou SH. Crizotinib: a novel and first-in-class multitargeted tyrosine kinase inhibitor for the treatment of anaplastic lymphoma kinase rearranged non-small cell lung cancer and beyond. Drug Des Devel Ther. 2011;5:471-485.

11. Moon YW, Park S, Sohn JH, et al. Clinical significance of progesterone receptor and HER2 status in estrogen receptor-positive, operable breast cancer with adjuvant tamoxifen. J Cancer Res Clin Oncol. 2011;137(7): 1123-1130.
12. National Comprehensive Cancer Network. NCCN Clinical Practice Guidelines in Oncology ${ }^{\mathrm{TM}}$ : Breast Cancer. Fort Washington, PA: National Comprehensive Cancer Network; 2011.

13. Scartozzi M, Bearzi I, Berardi R, Mandolesi A, Fabris G, Cascinu S. Epidermal growth factor receptor (EGFR) status in primary colorectal tumors does not correlate with EGFR expression in related metastatic sites: implications for treatment with EGFR-targeted monoclonal antibodies. J Clin Oncol. 2004;22(23):4772-4778.

14. Zidan J, Dashkovsky I, Stayerman C, Basher W, Cozacov C, Hadary A. Comparison of HER-2 overexpression in primary breast cancer and metastatic sites and its effect on biological targeting therapy of metastatic disease. Br J Cancer. 2005;93(5):552-556.

15. Sirera R, Bremnes RM, Cabrera A, et al. Circulating DNA is a useful prognostic factor in patients with advanced non-small cell lung cancer. J Thorac Oncol. 2011;6(2):286-290.

16. Zhao X, Han RB, Zhao J, et al. Comparison of epidermal growth factor receptor mutation statuses in tissue and plasma in stage I-IV non-small cell lung cancer patients. Respiration. 2013;85(2):119-125.

17. Mankoff DA. Molecular imaging to select cancer therapy and evaluate treatment response. Q J Nucl Med Mol Imaging. 2009;53(2): 181-192.

18. Blasberg RG, Tjuvajev JG. Molecular-genetic imaging: current and future perspectives. J Clin Invest. 2003;111(11):1620-1629.

19. Buck AK, Reske SN. Cellular origin and molecular mechanisms of 18F-FDG uptake: is there a contribution of the endothelium? $\mathrm{J} \mathrm{Nucl}$ Med. 2004;45(3):461-463.

20. Zander T, Scheffler M, Nogova L, et al. Early prediction of nonprogression in advanced non-small-cell lung cancer treated with erlotinib by using [(18)F]fluorodeoxyglucose and [(18)F]fluorothymidine positron emission tomography. J Clin Oncol. 2011;29(13): 1701-1708.

21. Kahraman D, Scheffler M, Zander T, et al. Quantitative analysis of response to treatment with erlotinib in advanced non-small cell lung cancer using 18F-FDG and 3'-deoxy-3'-18F-fluorothymidine PET. J Nucl Med. 2011;52(12):1871-1877.

22. Ullrich RT, Zander T, Neumaier B, et al. Early detection of erlotinib treatment response in NSCLC by $3^{\prime}$-deoxy-3'-[F]-fluoro-L-thymidine ([F]FLT) positron emission tomography (PET). PLoS ONE. 2008; 3(12):e3908

23. Yue J, Chen L, Cabrera AR, et al. Measuring tumor cell proliferation with 18F-FLT PET during radiotherapy of esophageal squamous cell carcinoma: a pilot clinical study. J Nucl Med. 2010;51(4): 528-534.

24. Scheffler M, Zander T, Nogova L, et al. Prognostic impact of [18F] fluorothymidine and [18F]fluoro-D-glucose baseline uptakes in patients with lung cancer treated first-line with erlotinib. PLOS ONE. 2013;8(1):e53081.

25. Kobe C, Scheffler M, Holstein A, et al. Predictive value of early and late residual $18 \mathrm{~F}$-fluorodeoxyglucose and $18 \mathrm{~F}$-fluorothymidine uptake using different SUV measurements in patients with non-small-cell lung cancer treated with erlotinib. Eur J Nucl Med Mol Imaging. 2012;39(7): 1117-1127.

26. Kahraman D, Holstein A, Scheffler M, et al. Tumor lesion glycolysis and tumor lesion proliferation for response prediction and prognostic differentiation in patients with advanced non-small cell lung cancer treated with erlotinib. Clin Nucl Med. 2012;37(11):1058-1064.

27. Wahl RL, Jacene H, Kasamon Y, Lodge MA. From RECIST to PERCIST: evolving considerations for PET response criteria in solid tumors. J Nucl Med. 2009;50 Suppl 1:122S-150S.

28. Wiehr S, von Ahsen O, Röse L, et al. Preclinical evaluation of a novel c-Met inhibitor in a gastric cancer xenograft model using small animal PET. Mol Imaging Biol. 2013;15(2):203-211.

29. Kasper B, Dimitrakopoulou-Strauss A, Pilz LR, Strauss LG, Sachpekidis C, Hohenberger P. Positron emission tomography as a surrogate marker for evaluation of treatment response in patients with desmoid tumors under therapy with imatinib. Biomed Res Int. 2013;2013:389672. 
30. Solit DB, Santos E, Pratilas CA, et al. $3^{\prime}$-deoxy-3'-[18F]fluorothymidine positron emission tomography is a sensitive method for imaging the response of BRAF-dependent tumors to MEK inhibition. Cancer Res. 2007;67(23):11463-11469.

31. Waldherr C, Mellinghoff IK, Tran C, et al. Monitoring antiproliferative responses to kinase inhibitor therapy in mice with $3^{\prime}$-deoxy-3'-18Ffluorothymidine PET. $J$ Nucl Med. 2005;46(1):114-120.

32. Krohn KA, Link JM, Mason RP. Molecular imaging of hypoxia. J Nucl Med. 2008;49 Suppl 2:129S-148S.

33. Hendrickson K, Phillips M, Smith W, Peterson L, Krohn K, Rajendran J. Hypoxia imaging with [F-18] FMISO-PET in head and neck cancer: potential for guiding intensity modulated radiation therapy in overcoming hypoxia-induced treatment resistance. Radiother Oncol. 2011;101(3): 369-375.

34. Li Z, Chu T. Recent advances on radionuclide labeled hypoxia-imaging agents. Curr Pharm Des. 2012;18(8):1084-1097.

35. Mileshkin L, Hicks RJ, Hughes BG, et al. Changes in $18 \mathrm{~F}-$ fluorodeoxyglucose and $18 \mathrm{~F}$-fluorodeoxythymidine positron emission tomography imaging in patients with non-small cell lung cancer treated with erlotinib. Clin Cancer Res. 2011;17(10):3304-3315.

36. Takahashi R, Hirata H, Tachibana I, et al. Early [18F]fluorodeoxyglucose positron emission tomography at two days of gefitinib treatment predicts clinical outcome in patients with adenocarcinoma of the lung. Clin Cancer Res. 2012;18(1):220-228.

37. Bengtsson T, Hicks RJ, Peterson A, Port RE. 18F-FDG PET as a surrogate biomarker in non-small cell lung cancer treated with erlotinib: newly identified lesions are more informative than standardized uptake value. $J$ Nucl Med. 2012;53(4):530-537.

38. Massoud TF, Gambhir SS. Molecular imaging in living subjects: seeing fundamental biological processes in a new light. Genes Dev. 2003;17(5):545-580.

39. van Dongen GA, Poot AJ, Vugts DJ. PET imaging with radiolabeled antibodies and tyrosine kinase inhibitors: immuno-PET and TKI-PET. Tumour Biol. 2012;33(3):607-615.

40. Tijink BM, Perk LR, Budde M, et al. (124)I-L19-SIP for immunoPET imaging of tumour vasculature and guidance of (131) I-L19-SIP radioimmunotherapy. Eur J Nucl Med Mol Imaging. 2009;36(8):1235-1244.

41. Verel I, Visser GW, Boellaard R, Stigter-van Walsum M, Snow GB, van Dongen GA. 89Zr immuno-PET: comprehensive procedures for the production of $89 \mathrm{Zr}$-labeled monoclonal antibodies. $J$ Nucl Med. 2003;44(8):1271-1281.

42. Dijkers EC, Oude MunninkTH, Kosterink JG, et al. Biodistribution of 89Zrtrastuzumab and PET imaging of HER2-positive lesions in patients with metastatic breast cancer. Clin Pharmacol Ther. 2010;87(5):586-592.

43. van der Bilt AR, Terwisscha van Scheltinga AG, Timmer-Bosscha $H$, et al. Measurement of tumor VEGF-A levels with 89Zr-bevacizumab PET as an early biomarker for the antiangiogenic effect of everolimus treatment in an ovarian cancer xenograft model. Clin Cancer Res. 2012;18(22):6306-6314.

44. Smith-Jones PM, Solit D, Afroze F, Rosen N, Larson SM. Early tumor response to Hsp90 therapy using HER2 PET: comparison with 18F-FDG PET. J Nucl Med. 2006;47(5):793-796.

45. Kenanova V, Wu AM. Tailoring antibodies for radionuclide delivery. Expert Opin Drug Deliv. 2006;3(1):53-70.

46. Cuartero-Plaza A, Martínez-Miralles E, Rosell R, Vadell-Nadal C, Farré M, Real FX. Radiolocalization of squamous lung carcinoma with 131Ilabeled epidermal growth factor. Clin Cancer Res. 1996;2(1):13-20.

47. Zhang $\mathrm{Y}$, Hong $\mathrm{H}$, Niu G, et al. Positron emission tomography imaging of vascular endothelial growth factor receptor expression with (61)Cu-labeled lysine-tagged VEGF(121). Mol Pharm. Epub November 16, 2012.

48. Gelovani JG. Molecular imaging of epidermal growth factor receptor expression-activity at the kinase level in tumors with positron emission tomography. Cancer Metastasis Rev. 2008;27(4):645-653.

49. Memon AA, Weber B, Winterdahl M, et al. PET imaging of patients with non-small cell lung cancer employing an EGF receptor targeting drug as tracer. Br J Cancer. 2011;105(12):1850-1855.
50. Meng X, Loo BW Jr, Ma L, Murphy JD, Sun X, Yu J. Molecular imaging with 11C-PD153035 PET/CT predicts survival in non-small cell lung cancer treated with EGFR-TKI: a pilot study. J Nucl Med. 2011;52(10): 1573-1579.

51. Slobbe P, Poot AJ, Windhorst AD, van Dongen GA. PET imaging with small-molecule tyrosine kinase inhibitors: TKI-PET. Drug Discov Today. 2012;17(21-22):1175-1187.

52. Miao Z, Levi J, Cheng Z. Protein scaffold-based molecular probes for cancer molecular imaging. Amino Acids. 2011;41(5):1037-1047.

53. Orlova A, Magnusson M, Eriksson TL, et al. Tumor imaging using a picomolar affinity HER2 binding affibody molecule. Cancer Res. 2006;66(8):4339-4348.

54. Friedman M, Orlova A, Johansson E, et al. Directed evolution to low nanomolar affinity of a tumor-targeting epidermal growth factor receptorbinding affibody molecule. $J$ Mol Biol. 2008;376(5):1388-1402.

55. Lindborg M, Cortez E, Höidén-Guthenberg I, et al. Engineered highaffinity affibody molecules targeting platelet-derived growth factor receptor $\beta$ in vivo. $J$ Mol Biol. 2011;407(2):298-315.

56. Miao Z, Ren G, Liu H, Jiang L, Cheng Z. Small-animal PET imaging of human epidermal growth factor receptor positive tumor with a $64 \mathrm{Cu}$ labeled affibody protein. Bioconjug Chem. 2010;21(5):947-954.

57. Miao Z, Ren G, Liu H, Jiang L, Cheng Z. Cy5.5-labeled Affibody molecule for near-infrared fluorescent optical imaging of epidermal growth factor receptor positive tumors. J Biomed Opt. 2010;15(3):036007.

58. Orlova A, Tolmachev V, Pehrson R, et al. Synthetic affibody molecules: a novel class of affinity ligands for molecular imaging of HER2expressing malignant tumors. Cancer Res. 2007;67(5):2178-2186.

59. Kramer-Marek G, Kiesewetter DO, Capala J. Changes in HER2 expression in breast cancer xenografts after therapy can be quantified using PET and (18)F-labeled affibody molecules. J Nucl Med. 2009;50(7):1131-1139.

60. Caravan P, Ellison JJ, McMurry TJ, Lauffer RB. Gadolinium(III) chelates as MRI contrast agents: structure, dynamics, and applications. Chem Rev. 1999;99(9):2293-2352.

61. Sipkins DA, Cheresh DA, Kazemi MR, Nevin LM, Bednarski MD, Li KC. Detection of tumor angiogenesis in vivo by alphaVbeta3-targeted magnetic resonance imaging. Nat Med. 1998;4(5):623-626.

62. Boles KS, Schmieder AH, Koch AW, et al. MR angiogenesis imaging with Robo4- vs alphaVbeta3-targeted nanoparticles in a B16/F10 mouse melanoma model. FASEB J. 2010;24(11):4262-4270.

63. Mulder WJ, Strijkers GJ, van Tilborg GA, Griffioen AW, Nicolay K. Lipid-based nanoparticles for contrast-enhanced MRI and molecular imaging. NMR Biomed. 2006;19(1):142-164.

64. Mulder WJ, van der Schaft DW, Hautvast PA, et al. Early in vivo assessment of angiostatic therapy efficacy by molecular MRI. FASEB J. 2007;21(2):378-383.

65. Guimaraes A R, Ross R, Figuereido J L, Waterman P, Weissleder R. MRI with magnetic nanoparticles monitors downstream anti-angiogenic effects of mTOR inhibition. Mol Imaging Biol. 2011;13:314-320.

66. Abdolahi M, Shahbazi-Gahrouei D, Laurent S, et al. Synthesis and in vitro evaluation of MR molecular imaging probes using J591 mAbconjugated SPIONs for specific detection of prostate cancer. Contrast Media Mol Imaging. 2013; 8:175-184.

67. Ward KM, Aletras AH, Balaban RS. A new class of contrast agents for MRI based on proton chemical exchange dependent saturation transfer (CEST). J Magn Reson. 2000;143(1):79-87.

68. Liu G, Liang Y, Bar-Shir A, et al. Monitoring enzyme activity using a diamagnetic chemical exchange saturation transfer magnetic resonance imaging contrast agent. J Am Chem Soc. 2011;133(41): $16326-16329$

69. Gilad AA, van Laarhoven HW, McMahon MT, et al. Feasibility of concurrent dual contrast enhancement using CEST contrast agents and superparamagnetic iron oxide particles. Magn Reson Med. 2009;61(4): 970-974.

70. Terreno E, Barge A, Beltrami L, et al. Highly shifted LIPOCEST agents based on the encapsulation of neutral polynuclear paramagnetic shift reagents. Chem Commun (Camb). 2008:600-602. 
71. Glunde K, Bhujwalla ZM. Metabolic tumor imaging using magnetic resonance spectroscopy. Semin Oncol. 2011;38(1):26-41.

72. Beloueche-Babari M, Jackson LE, Al-Saffar NM, Workman P, Leach MO, Ronen SM. Magnetic resonance spectroscopy monitoring of mitogen-activated protein kinase signaling inhibition. Cancer Res. 2005;65(8):3356-3363.

73. Klawitter J, Anderson N, Klawitter J, et al. Time-dependent effects of imatinib in human leukaemia cells: a kinetic NMR-profiling study. Br J Cancer. 2009;100(6):923-931.

74. Sankaranarayanapillai M, Tong WP, Yuan Q, et al. Monitoring histone deacetylase inhibition in vivo: noninvasive magnetic resonance spectroscopy method. Mol Imaging. 2008;7(2):92-100.

75. Kato Y, Okollie B, Artemov D. Noninvasive $1 \mathrm{H} / 13 \mathrm{C}$ magnetic resonance spectroscopic imaging of the intratumoral distribution of temozolomide. Magn Reson Med. 2006;55(4):755-761.

76. Howe FA, Robinson SP, Rodrigues LM, Griffiths JR. Flow and oxygenation dependent (FLOOD) contrast MR imaging to monitor the response of rat tumors to carbogen breathing. Magn Reson Imaging. 1999;17(9):1307-1318.

77. Unnikrishnan S, Klibanov AL. Microbubbles as ultrasound contrast agents for molecular imaging: preparation and application. AJR Am J Roentgenol. 2012;199(2):292-299.

78. Anderson $\mathrm{CR}, \mathrm{Hu} \mathrm{X}$, Zhang $\mathrm{H}$, et al. Ultrasound molecular imaging of tumor angiogenesis with an integrin targeted microbubble contrast agent. Invest Radiol. 2011;46(4):215-224.

79. Willmann JK, Kimura RH, Deshpande N, Lutz AM, Cochran JR, Gambhir SS. Targeted contrast-enhanced ultrasound imaging of tumor angiogenesis with contrast microbubbles conjugated to integrin-binding knottin peptides. J Nucl Med. 2010;51(3):433-440.
80. Willmann JK, Paulmurugan R, Chen K, et al. US imaging of tumor angiogenesis with microbubbles targeted to vascular endothelial growth factor receptor type 2 in mice. Radiology. 2008;246(2):508-518.

81. Anderson CR, Rychak JJ, Backer M, Backer J, Ley K, Klibanov AL. scVEGF microbubble ultrasound contrast agents: a novel probe for ultrasound molecular imaging of tumor angiogenesis. Invest Radiol. 2010;45(10):579-585.

82. Warram JM, Sorace AG, Saini R, Umphrey HR, Zinn KR, Hoyt K. A triple-targeted ultrasound contrast agent provides improved localization to tumor vasculature. J Ultrasound Med. 2011;30(7):921-931.

83. Deshpande N, Ren Y, Foygel K, Rosenberg J,Willmann J K. Tumor angiogenic marker expression levels during tumor growth: longitudinal assessment with molecularly targeted microbubbles and US imaging. Radiology. 2011; 258: 804-811.

84. Rabin O, Manuel Perez J, Grimm J, Wojtkiewicz G, Weissleder R. An $\mathrm{X}$-ray computed tomography imaging agent based on long-circulating bismuth sulphide nanoparticles. Nat Mater. 2006;5(2):118-122.

85. Hyafil F, Cornily JC, Feig JE, et al. Noninvasive detection of macrophages using a nanoparticulate contrast agent for computed tomography. Nat Med. 2007;13(5):636-641.

86. Jackson PA, Rahman WN, Wong CJ, Ackerly T, Geso M. Potential dependent superiority of gold nanoparticles in comparison to iodinated contrast agents. Eur J Radiol. 2010;75(1):104-109.

87. Aydogan B, Li J, Rajh T, et al. AuNP-DG: deoxyglucose-labeled gold nanoparticles as X-ray computed tomography contrast agents for cancer imaging. Mol Imaging Biol. 2010;12(5):463-467.
International Journal of Nanomedicine

\section{Publish your work in this journal}

The International Journal of Nanomedicine is an international, peerreviewed journal focusing on the application of nanotechnology in diagnostics, therapeutics, and drug delivery systems throughou the biomedical field. This journal is indexed on PubMed Central, MedLine, CAS, SciSearch ${ }^{\circledR}$, Current Contents ${ }^{\circledR} /$ Clinical Medicine,

\section{Dovepress}

Journal Citation Reports/Science Edition, EMBase, Scopus and the Elsevier Bibliographic databases. The manuscript management system is completely online and includes a very quick and fair peer-review system, which is all easy to use. Visit http://www.dovepress.com/ testimonials.php to read real quotes from published authors. 